\title{
LA REPRESENTACIÓN DE LOS Y LAS INDÍGENAS EN LA ENSEÑANZA DE LA HISTORIA EN LA EDUCACIÓN BÁSICA CHILENA. EL CASO DE LOS TEXTOS DE ESTUDIO DE HISTORIA DE CHILE
}

\author{
REPRESENTATION OF INDIGENOUS PEOPLE IN THE TEACHING \\ OF HISTORY IN PRIMARY EDUCATION IN CHILE. \\ CASE STUDY OF TEXTS OF HISTORY OF CHILE
}

\author{
Gabriel Villalón Gálvez* y Joan Pagès Blanch**
}

\begin{abstract}
Este artículo analiza la presencia de los y las indígenas en la enseñanza de la historia en la educación básica en Chile. Para esto revisa tres textos de estudio de educación básica entregados por el Ministerio de Educación de Chile para la asignatura de Historia, Geografía y Ciencias Sociales en los que se desarrollan los contenidos de historia de Chile. Los resultados muestran que los y las indígenas están incorporados en los contenidos de historia de Chile. Y que las representaciones sobre estos se asocian al mestizaje y las tradiciones foklóricas y a la imagen del guerrero y rebelde, lo que no cambia los prejuicios y estereotipos construidos respecto del indígena en Chile.
\end{abstract}

Palabras claves: Indígenas, enseñanza de la historia, textos de estudio

This article analyzes the presence of indigenous men and women in the teaching of history in primary education in Chile. For this study reviews three texts of basic education provided by the Ministry of Education of Chile to the subject of History, Geography and Social Sciences in which the contents of Chilean history unfold. The results show that indigenous men and women are incorporated in the content of Chilean history. And that representations of these are associated with mixing and fokloricas traditions and image of the warrior and rebel, which does not change the prejudices and stereotypes regarding indigenous constructed in Chile.

Key words: indigenous, history teaching, textbook.

\section{Introducción}

El novelista noruego Mesbo relata en una de sus novelas que:

La presencia de los aborígenes en la vida social del país es prácticamente nula, y solo se oye hablar de ellos en los debates públicos que giran alrededor de su cultura, sus intereses y su idiosincrasia. Muchos australianos cuelgan obras de arte aborigen en las paredes de su casa como si de esta manera ya pagasen peaje por su hipocresía. Pero, en realidad, los lugares donde los aborígenes estamos más bien representados es en las colas de la seguridad social, en las estadísticas de suicidio y en las cárceles. Si eres aborigen, la probabilidad de que vayas a parar a la cárcel es veintiséis veces más alta que para cualquier otro australiano (Mesbo, 2015:23).

El lugar de los aborígenes, o de los indígenas, en el currículo escolar es una consecuencia del lugar que ocupan en la sociedad como refleja el escritor noruego Mesbo en su novela. Esta situación arranca desde el mismo momento de la ocupación y la posterior colonización por parte de los europeos en América, Asia, África u Oceanía. En algunos lugares se soluciona con las independencias, pero no es el caso ni de América ni de Oceanía ni de ningún país en el que las independencias estuvieron protagonizadas por los descendientes de los europeos. Los indígenas, los aborígenes siguieron excluidos y marginados del poder como señala Arcos en el caso de México y, en concreto, de Chiapas:

\footnotetext{
* Universidad de Tarapacá, Departamento de Ciencias Históricas y Geográficas, Arica, Chile. Correo electrónico: gvillalon@ uta.cl

** Universidad Autónoma de Barcelona, Departamento de Didáctica de la Lengua, la Literatura y las Ciencias Sociales, Bellaterra, España. Correo electrónico: joan.pages@uab.cat
} 
Cuando los europeos entraron en contacto con las sociedades originarias del continente americano, les asignaron el nombre de "indígenas" o "indios" a los diversos pueblos que mantenían identidades propias y culturas específicas; una cultura diferente e inferior desde el punto de vista racial, cultural, lingüístico y científico. De tal forma, la diversidad étnica de todos los habitantes quedó sesgada y sin posibilidades de desarrollo propio y evolución" (Arcos, 2010:122).

La situación de exclusión no ha variado mucho. Sí, en cambio, se han modificado, a veces sustancialmente, sus tradiciones y sus costumbres, ya que muchos pueblos indígenas fueron obligados a abandonar sus lugares históricos en aras del progreso y se vieron abocados a vivir en unos contextos donde difícilmente las pudieron mantener vivas. Para Arcos:

El indígena de hoy ha sufrido cambios y transformaciones, por lo que se puede encontrar diferencias en comparación con los de la época colonial, incluso, hasta hace 60 años. Sin embargo, a pesar de los cambios, mantiene ciertos rasgos culturales de su origen sin renunciar a su identidad, misma que se expresa en formas de vida comunitaria, comunicación oral a través de su lengua madre, relación con la naturaleza, una estructura propia de organización social, medicina y una sabiduría ancestral (ciencias indígenas) (Arcos, 2010: 126).

¿Qué deberíamos hacer desde el currículo y la enseñanza de la historia y de las ciencias sociales para hacer visible la presencia de los indígenas, de sus historias, de sus tradiciones, costumbres y creencias?, ¿qué relación debería existir entre la historia nacional hoy en vigor en la mayor parte de los países del mundo y la historia de aquellos colectivos y grupos humanos, como los indígenas, que, además de marginados, han sido ignorados en los textos y en las prácticas?

Nuestro propósito en este trabajo es analizar la situación de los indígenas en el currículo escolar chileno en el caso de los textos de estudio de educación básica que tratan los contenidos de historia de Chile.

\section{La Historia escolar, la enseñanza de la Historia y la presencia de los subalternos}

Las representaciones que se realizan sobre el pasado son variadas, en cuanto a los relatos históricos podemos indicar que son de tres tipos. En primer lugar tenemos la historia que se aprende en los currículos escolares; luego la historia cotidiana, que se presenta como un componente de la memoria colectiva que articula los recuerdos, relatos de una sociedad o grupo humano constituyendo una serie de valores y creencias que les otorgan identidad; finalmente contamos con la historia académica, que se refiere a la ciencia que desarrollan los historiadores o científicos sociales (Carretero, 2007; 36). Estos tres tipos de relatos históricos deben ser entendidos como narraciones que se encuentran interrelacionadas entre sí. Por ejemplo, la historia escolar se nutre de la historia académica para construir sus contenidos y construye narraciones que luego son utilizadas por los relatos cotidianos de la historia (Carretero, 2007). Entre estos tres tipos de relatos destaca el de la historia escolar que de acuerdo con Carretero (2007) incluye una gran cantidad de valores que se enlazan en una trama de relatos cuya finalidad tradicionalmente ha sido la formación en los estudiantes de una visión positiva de la identidad de su nación. La escuela tradicionalmente en el siglo XIX y en buena parte del siglo XX ha transmitido un relato de la historia que tiene como finalidad principal la construcción de ciudadanos obedientes y comprometidos con la patria (Pagès, 1994).

En el siglo XX y hoy ya en el siglo XXI podemos identificar que la historia escolar en la práctica se ha traducido en distintos tipos de currículos que se diferencian de acuerdo con sus finalidades en relación con lo que quieren conseguir en relación a la formación de los estudiantes. Tradicionalmente se han descrito tres tipos de currículos de la enseñanza de la historia, técnico o positivista, humanista o práctica y la tradición crítica (Benejam y Pagès, 2004; Pagès, 1994; Santisteban y Pagès, 2011).

El currículo técnico o positivista ha sido por definición el modelo dominante en la historia escolar, entre sus características se encuentran una concepción conservadora de la sociedad, el mantenimiento del statu quo y el desarrollo del nacionalismo. En este modelo de la enseñanza de la historia se ha privilegiado la reproducción del orden social, se entiende que se debe formar buenos ciudadanos, 
estos son aquellos a los que el Estado o los grupos dominantes les otorgan esa categoría por aceptar sin cuestionamientos las "reglas del juego" (Pagès, 1994). En la práctica este currículum ha construido discursos universales que entienden a la sociedad como un cuerpo homogéneo, en donde la diversidad y la diferencia son invisibilizados. La enseñanza de la historia en estos marcos está centrada en un aprendizaje memorístico, en que el estudiante domina el contenido sustantivo de la historia que se representa en los grandes hechos y hombres de la historia nacional (Levesque, 2010).

Los relatos escolares de la historia han negado desde el enfoque descrito el tratamiento de los conflictos y la historicidad de los grupos subalternos, por ejemplo como indican en su investigación Sarah B. Shear, Ryan T. Knowles, Gregory J. Soden y Antonio J. Castro (2015) los discursos tradicionales de la enseñanza de la historia han silenciado las experiencias históricas de las minorías en Estados Unidos, esto ha traído como consecuencia que los relatos de los currículos de historia hayan negado continuamente los actos de xenofobia y discriminación. Sarah B. Shear y sus colegas (2015) muestran además que los modelos tradicionales de la enseñanza de la historia han negado el aporte de los grupos minoritarios en el desarrollo histórico de los Estados Unidos, es así como el tratamiento de contenidos asociados a los grupos de emigrantes latinos o el papel de los grupos indígenas ha sido minimizado. En una línea similar Luis Urieta (2004) ha indicado que pese a que los esfuerzos por desarrollar planes de estudio de la historia que buscan construir una ciudadanía que se haga cargo de la diversidad y de las características multiculturales de la sociedad actual, en el caso norteamericano, los discursos tradicionales de la enseñanza han continuado dominando la práctica, lo que se ha traducido en que en las escuelas se continúe transmitiendo una idea de que "la ciudadanía completa" norteamericana está reservada para el hombre blanco.

La ausencia de temáticas asociadas a grupos subalternos que permita que los estudiantes puedan aprender para la vida en una sociedad diversa e intercultural ha sido una característica de la enseñanza de la historia, como hemos visto en una investigación anterior, no es habitual que en los manuales de didáctica de la historia, aun en los más recientes, figure un capítulo dedicado a los actores, a los protagonistas del devenir histórico o a los personajes y grupos invisibles. El predominio de la historia eurocéntrica y androcéntrica, de los relatos nacionales y de la tradición, es tan abrumador que ni siquiera quienes reivindican cambios en la enseñanza y en la formación del profesorado han planteado este debate y han elaborado estrategias para que la historia deje de estar protagonizada por las minorías dirigentes y se abra a la gente común, a los otros, a los diferentes (Villalón y Pagès, 2013). La revisión de los textos de estudio de historia de la educación primaria chilena nos muestra que se ha incorporado la historia de los otros y otras. Sin embargo, siguen existiendo silencios en la escuela sobre algunos grupos como los homosexuales que no aparecen en ninguna propuesta. La integración de la historia de los otros y otras no significa un cambio en la manera de concebir la construcción del relato histórico en los textos ni en la enseñanza y el aprendizaje. La inclusión de los otros y otras ha sido de tipo periférico y como un anexo a la historia oficial que continúa dominando la manera de ordenar la narración histórica en los textos de estudio. El relato que se hace en los textos sobre los otros y las otras en ocasiones niega su historicidad y es construido desde una visión dominante, desde arriba (Villalón y Pagès, 2013; 135).

\section{La representación de la historicidad de los grupos indígenas en la enseñanza de la historia}

Los y las indígenas han sido uno de los grupos subalternos que la enseñanza de la historia tradicional ha negado o invisibilizado. Las investigaciones sobre el currículum estadounidense han mostrado que la historia escolar ha ignorado los episodios oscuros relacionados con la destrucción de las culturas indígenas (Fixico, 1998). Las representaciones de los indígenas en los textos de estudio ha consolidado los estereotipos y prejuicios sobre la población indígena, ya que ha presentado una imagen de ladrones, borrachos, salvajes y perezosos (Shear et al., 2015). En las conclusiones de su investigación sobre los estándares y textos de estudio en Estados Unidos Shear y sus colegas nos indican que la incorporación de los temas asociados a las culturas indígenas continúa estando centrada en contenidos que hablan de una historia lejana antes del 1900 y construida con narrativa propias de la historia americana-eurocéntrica (Shear et al., 2015). 
Luis Urrieta (2004) indica que en la escuela se ha construido un discurso que ha validado la subyugación del indígena, esto mediante la transmisión de relatos que dan cuentan de la supremacía blanca y los principios cristianos de "la salvación" y la construcción de una imagen del indígena como un ser que necesita ser salvado y civilizado. Urrieta en su investigación concluye que la enseñanza de la historia sobre los indígenas está marcada por la invisibilidad y por un aprendizaje acrítico. Por acrítica, se refiere a las representaciones que se centraron en el estereotipo y el enfoque de festivo a aprender acerca de un / otro en los Estados Unidos, esto, según Banks (2003), son las "contribuciones enfoque", que se centra por lo general solo en héroes, días de fiesta, y otros elementos culturales discretos de multiculturalismo (Urrieta, 2004).

Dosil Mancilla (2010) en su revisión sobre la construcción de la identidad indígena en la enseñanza de la historia en México, indica que pese a las iniciativas desde las autoridades por desarrollar un currículo que incorpore la interculturalidad esos intentos no han fructificado, ya que no consideran las demandas de las comunidades indígenas. Dosil Mancilla (2010) indica que la enseñanza intercultural ha sido instalada en las escuelas indígenas desconociendo la tradición de educación intercultural que ha sido desarrollada por estas comunidades desde siempre. En cambio los planes educativos en las zonas urbanas la escuela no ha considerado la interculturalidad, esto ha tenido como consecuencia que los maestros y los estudiantes mestizos identifiquen por lo general la realidad indígena con la ignorancia y la pobreza. A esto se suma que los maestros al enseñar reducen los contenidos asociados a las temáticas indígenas, los reducen a relatar aspectos folklóricos (vestimentas, danzas, comidas, etc.) y a una representación de que estos grupos están destinados a desaparecer en el México moderno. En cuanto a los materiales didácticos, tomando como ejemplo los textos de estudio, indica que se centran en mostrar el léxico de actividades cotidianas de las comunidades indígenas, aspectos folklóricos y vinculadas a la identidad nacional; y una idea de una sociedad sin conflictos en donde los distintos grupos culturales y étnicos no tienen problemas para convivir, en que la desigualdad social y económica no existe y son sujetos de derecho en igualdad de condiciones (Mancilla, 2010).

La revisión que hemos realizado nos permite establecer que la enseñanza de la historia dominada por concepciones técnicas del currículum ha creado una narración del indígena que se caracteriza por la negación de su historicidad, un análisis desde los marcos de la historia eurocéntrica, una negación de los conflictos asociados a la identidad indígena en la sociedad actual y un relato ubicado temporalmente en el pasado o centrado en aspectos folklóricos de la vida indígena.

\section{La representación de lo indígena en los textos de estudio de historia de Chile en la educación primaria}

Tomando en cuenta lo anterior en este artículo hemos querido revisar cuáles son las representaciones de lo indígena en la enseñanza de la historia en Chile, para esto nos hemos centrado en el análisis de los textos de estudio de historia de Chile utilizados en la educación primaria.

Para el desarrollo de este texto nos hemos planteado las siguientes preguntas: ¿Cuál es la presencia que tienen los indígenas en los textos de estudio de historia de Chile para la educación primaria? Y ¿cómo son las narraciones sobre los indígenas en los textos de estudio de historia de Chile para la educación primaria?

El análisis que hemos realizado ha seguido los marcos de la metodología cualitativa. La investigación cualitativa se plantea el estudio de la realidad por lo que para investigar debemos instalarnos en el contexto natural, intentando sacar sentido o interpretar los fenómenos de acuerdo con los significados que tienen (Rodríguez, Gil y García, 1999). Para el análisis hemos utilizado el análisis de contenido y documentos (J. Mckernan, 2008).

Para el análisis hemos tomado como muestra los textos de estudio de historia para la educación general básica en Chile. Entre estos textos hemos elegidos tres que respondieron a dos criterios: 1) ser los textos distribuidos por el Ministerio de Educación de Chile, 2) desarrollar los contenidos referidos a la historia de Chile. Los textos seleccionados fueron: Historia, Geografía y Ciencias Sociales para $2^{\circ}$ año básico de la Editorial Santillana publicado en 2012; los textos de estudio de Historia, Geografía y Ciencias Sociales de $5^{\circ}$ y $6^{\circ}$ año básico de la editorial Zig-Zag publicado en 2012.

A continuación describimos los resultados del análisis realizado, para esto primero nos referiremos a la presencia que tienen los indígenas en los contenidos de historia de Chile y en segundo lugar, 
describiremos las representaciones de los indígenas en los textos de estudio.

\section{Presencia de los indígenas en la enseñanza de la historia de Chile en la educación primaria}

La enseñanza de la historia de Chile se ocupa en el currículum chileno de los contenidos de tres cursos, segundo año básico, quinto año básico y sexto año básico. El tratamiento de los contenidos de historia de Chile en los textos escolares está planteado desde una visión tradicional ordenada cronológicamente, en concordancia con esto, los contenidos por curso se ordenan de la siguiente manera: $2^{\circ}$ año básico tratamiento de los contenidos asociados a las culturas originarias prehispánicas en Chile; $5^{\circ}$ año básico tratamiento de los contenidos asociados a la historia de Chile para el período de conquista y colonia; y $6^{\circ}$ año básico tratamiento de los contenidos desde la independencia hasta la historia reciente de Chile.

Los indígenas aparecen con presencia en los tres cursos y tres textos analizados. En el caso del $2^{\circ}$ año básico los indígenas tienen presencia en 10 de los temas desarrollados por el texto de estudio para enseñar la historia de Chile. En el caso del texto de estudio de $5^{\circ}$ año básico los indígenas tienen presencia en 22 de los temas desarrollados. Para el caso del $6^{\circ}$ año básico los indígenas tienen presencia en siete temas de los desarrollados para la enseñanza de la historia de Chile.

En términos cronológicos las temáticas asociadas a los indígenas en la historia de Chile se concentran en los contenidos asociados a las culturas originarias o Chile prehispánico y a los contenidos referidos al período de conquista y colonia. De los 39 temas asociados a los indígenas el $82 \%$ está vinculado a temáticas referidas al período pre-hispánico, la conquista o la colonia. El 18\% restante se asocia a contenidos que se desarrollan en el $6^{\circ}$ año básico en temas referidos al siglo XIX. Una cuestión que llama la atención es que para los contenidos referidos a la historia del siglo XX y la historia reciente de Chile la invisibilidad de los y las indígenas es absoluto, es así como contenidos referidos a los conflictos indígenas como el problema mapuche por ejemplo no son tomados en cuenta (Zig-Zag, 2012b).

La presencia indígena en la enseñanza de la historia de Chile aparece relacionada a distintas temáticas. En el caso del libro de $2^{\circ}$ año básico (Santillana, 2012) hemos identificado que los contenidos referidos a los indígenas se relacionan en primer lugar con el concepto de pueblos originarios. El texto desarrolla cinco lecciones asociadas con este concepto. En cada una de estas lecciones el tema de los pueblos originarios se asocia a los conceptos de organización social y económica, formas de vida y ubicación geográfica (Santillana, 2012). El segundo concepto sobre el que asocia el tratamiento de contenidos referidos a los indígenas en el texto de segundo año medio es el de mestizaje. El concepto de mestizaje por su parte aparece relacionado con conceptos como tradiciones culturales y sincretismo (Santillana, 2012).

En el caso del texto de estudio de $5^{\circ}$ año básico la presencia indígena se asocia fundamentalmente con el período de conquista y colonia. Los temas asociados a los indígenas se asocian en primer lugar con los conceptos de resistencia, es así como entre los veintidós contenidos asociados a los indígenas nos encontramos con ocho menciones $36 \%$ asociado al concepto de resistencia indígena o guerra (Zig-Zag, 2012a). El segundo concepto con el que se asocian los contenidos sobre los indígenas en el curso de $5^{\circ}$ año básico es el de las relaciones fronterizas, el que es mencionado en cinco ocasiones concentrando el 22,7\%. Los contenidos restantes en el texto de $5^{\circ}$ se distribuyen entre los conceptos de trabajo indígena y mestizaje cada uno concentrando el $18 \%$. Es interesante hacer notar que en el caso del $5^{\circ}$ año básico se hace referencia a temas como los derechos indígenas, la discriminación y la vida de las mujeres indígenas (Zig-Zag, 2012a).

El texto de estudio de $6^{\circ}$ año básico es el que concentra menos presencia de contenidos que hacen referencia a los indígenas, de las siete menciones que hemos identificado se distribuyen en dos menciones que hacen alusión a la invisibilidad de los indígenas, a la participación en las montoneras, a los relatos de viajeros, a los derechos para los indígenas y a la estructura social.

La revisión de los textos de estudio nos permite establecer que los contenidos referidos a los indígenas se encuentran presente en los tres textos concentrándose en los contenidos relacionados con el curso de $5^{\circ}$ básico. En términos cronológicos los temas indígenas aparecen asociados fundamentalmente a la historia del período de conquista y colonial; y se hacen invisibles al acercarnos a la historia de contemporánea o reciente. El tratamiento de la historia indígena se relaciona principalmente con los conceptos de pueblos originarios y formas 
de organización social y económica; el concepto de resistencia indígena y el concepto de mestizaje asociado al legado cultural desde una perspectiva folklórica.

\section{La representación de los indígenas en la enseñanza de la historia en los textos de estudio de Historia de Chile de educación básica}

La revisión de los textos de estudio de educación básica nos ha permitido identificar dos tipos de representaciones: a) el legado cultural mestizaje y el folklore nacional; b) El indígena como guerrero y rebelado.

\section{a) El legado cultural mestizaje y el folklore nacional}

Un primer tipo de relato que hemos identificado sobre la historicidad indígena presente en los textos de estudio es aquel que hace referencia a la relación entre el mestizaje y el legado cultural representado en la tradición o folklore nacional. Las bases de este relato se encuentran en primer lugar en el estudio de los indígenas por ser parte de los pueblos originarios. El libro de texto de $2^{\circ}$ año básico presenta de la siguiente manera el contenido referido a las culturas prehispánicas:

\begin{abstract}
Antes de la llegada de los españoles, cuando América no se dividía en países, en el territorio donde ahora se ubica Chile habitaron diversos pueblos. Hoy los llamamos pueblos originarios, por ser los primeros habitantes de estas tierras (Santillana, 2012:50).
\end{abstract}

El relato sobre los indígenas continúa con una narración de las características culturales generales (aspectos económicos, sociales, formas de vida y actividades cotidianas) para cada una de las culturas indígenas prehispánicas. Estos relatos buscan dar cuenta de las diferencias en las vidas de los pueblos originarios entre sí y con la realidad de los estudiantes, como podemos ver en el siguiente extracto del texto de $2^{\circ}$ año básico:

Estos pueblos tuvieron distintos modos de vida. Algunos desplazaban sus viviendas de un lugar a otro en busca de alimento, por medio de la caza, la pesca y la recolección de frutos. Estos pueblos tenían un modo de vida nómada. Como vestimenta, los pueblos nómadas utilizaban las pieles de los animales que cazaban y sus viviendas eran provisorias. Otros pueblos producían su propio alimento, dedicándose a la agricultura y la ganadería, por lo que no necesitaban desplazarse. Su modo de vida era sedentario (Santillana, 2012:50).

Una vez descritas las características de los pueblos originarios el relato comienza a dar cuenta de elementos de permanencia de estas culturas en el Chile actual por medio del uso del concepto de mestizaje y procesos afines. El mestizaje es descrito como un proceso resultado de las relaciones establecidas entre los indígenas y conquistadores. El mestizaje en los textos de estudio es descrito como un proceso ajeno al conflicto, dando a entender que la instalación de las tradiciones occidentales de los conquistadores sobre los y las indígenas habría sido un proceso pacífico y no impuesto. Por ejemplo podemos ver el relato que hace el texto de $2^{\circ}$ básico en relación con la llegada de los españoles y sus aportes culturales "Los españoles conquistaron gran parte del continente para luego vivir en él y traspasaron parte importante de sus costumbres, sus creencias y su cultura a los pueblos indígenas" (Santillana, 2012:78).

El relato del mestizaje como un proceso ajeno al conflicto es reafirmado por el texto cuando se refiere al surgimiento de los mestizos como grupo, al respecto indica que "Se formaron familias en que uno de los padres era español y el otro indígena. Los hijos que tuvieron tenían rasgos de ambos y se llamaron mestizos. La mayoría de los chilenos somos mestizos" (Santillana, 2012:78).

La negación de cualquier conflicto asociado al proceso de mestizaje es reafirmado por los textos cuando se refiere al aporte de los indígenas al mestizaje indicando que "A su vez, los pueblos originarios les mostraron a los españoles el maíz, la papa y sus preparaciones de comidas. También les enseñaron a domesticar animales como la llama y a curar enfermedades con plantas medicinales" (Santillana, 2012:79).

El mestizaje se representa como una de las manifestaciones sobre las cuales perviven las culturas originarias en la actualidad y por medio del cual entregan su aporte a la construcción de la identidad nacional. En el texto de $5^{\circ}$ año básico se da cuenta 
de esto tomando el concepto de sincretismo, por medio del cual se intenta explicar quedaron presentes las tradiciones indígenas en la cultura chilena:

Las creencias religiosas americanas no desaparecieron. La Iglesia debió aceptar la importancia de tradiciones y ritos nativos que se fueron fusionando con las expresiones religiosas católicas. Fue el caso, entre muchos otros, del culto a la Virgen María que los pueblos originarios asociaron con el culto a la Madre Tierra (Zig-Zag, 2012a:104).

El relato construido por los textos de estudio nos muestra que la pervivencia de lo indígena en el Chile además de mantenerse por el mestizaje, ha perdurado por la existencia de las tradiciones que mantienen los grupos indígenas en la actualidad. El relato que hacen los textos al respecto destaca por sobre todo la pervivencia de cuestiones folklóricas como bailes, ritos y comidas. Por ejemplo sobre los mapuches indica que:

El pueblo mapuche ha mantenido varias de sus tradiciones y ceremonias religiosas, como el machitún, que es una ceremonia para curar a los enfermos. Su arte de tejer lana es una de las más destacadas; y su lengua, el mapuzugun, también ha perdurado en el tiempo (Santillana, 2012:75).

Un discurso similar se hace por ejemplo en el caso de los rapa nui:

El pueblo rapa nui también ha conservado sus costumbres. Algunas de sus tradiciones que han permanecido son las ceremonias y fiestas, como el Tapati Rapa Nui, y también sus danzas, como el sau sau, el ula ula y el tamuré. Para realizar sus bailes utilizan trajes típicos con plumas y fibra de plátano. Sus tatuajes y los moais son otra expresión artística que los caracteriza y que han perdurado en el tiempo (Santillana, 2012:75).

\section{b) El indígena como guerrero y rebelado}

El segundo relato que aparece presente en los textos de estudio de educación básica es aquel que otorga al indígena el carácter de guerrero y rebelado. Se transmite hacia los estudiantes una concepción de las y los indígenas como violentos o guerreros culturalmente, se da a entender que la resistencia indígena a la conquista española es una cuestión cultural y no de reacción ante la invasión de su territorio.

Una de las narraciones que ejemplifican lo anterior son aquellos que muestran cómo los conquistadores españoles son víctimas del carácter agresivo y beligerante de los indígenas. Por ejemplo sobre el caso del fracaso de Diego de Almagro se indica que:

\begin{abstract}
Almagro decide abandonar Chile: el frío, la resistencia araucana, y la pobreza del territorio lo convencieron de abandonar su empresa. Rápidamente emprendió el regreso, esta vez por el desierto de Atacama, y no regresó nunca más a Chile (Zig-Zag, 2012a).
\end{abstract}

En la misma línea el texto de $5^{\circ}$ año indica que probablemente el principal problema que tuvieron los españoles en Chile fue la resistencia de los mapuches, al respecto indica que:

Los primeros años de la conquista los años que transcurrieron entre 1541 y 1549 , es decir, los primeros años de la conquista, fueron muy complicados para los conquistadores españoles. Las dificultades que experimentaron fueron de diverso tipo y se mencionan en el siguiente esquema. De todos los problemas, el más grave fue el de la rebeldía indígena. Uno de los episodios más violentos se vivió en Santiago, en 1541. Los picunches, dirigidos por el cacique Michimalonko, asaltaron la ciudad el 11 de septiembre de1541, hecho que terminó con la destrucción de la mayoría de las casas de Santiago (Zig-Zag, 2012a).

La representación del indígena como un guerrero y propenso a la rebelión es reafirmada de manera definitiva por el texto de $5^{\circ}$ año básico cuando establece que la resistencia de los mapuches a la conquista se encuentra en directa relación con su naturaleza guerrera como se puede desprender del siguiente apartado:

Fue hacia 1550, cuando los españoles decidieron avanzar hacia el Sur e ingresar a las tierras mapuches. Fundaron ciudades 
y comenzó una larga resistencia que los españoles jamás pudieron doblegar completamente. ¿Por qué resistieron los araucanos? Por rechazar la encomienda, y defender sus tierras y su libertad. ¿Por qué les costó tanto a los españoles? Porque enfrentaron a un pueblo acostumbrado a la guerra y porque carecían de jefe político. Hacer la paz con un lonco o cacique no implicaba la paz con todos los araucanos (Zig-Zag, 2012a:54).

En el párrafo anteriormente citado el texto explica la resistencia mapuche a cuestiones que se desprenden de su cultura guerrera y a las estructuras organizacionales que se convertía en un obstáculo para la paz y la colonización española. De esta manera el texto exime cualquier opción para que los y las estudiantes puedan desconstruir los prejuicios sobre el indígena como pueblo combativo que ha construido el relato cotidiano de la historia de Chile.

La imagen propensa a la violencia del indígena propuesta por los textos se mantiene en los relatos que se hacen en relación con la sociedad fronteriza, a propósito de la organización de los Parlamentos para establecer acuerdos de Paz, el texto de $5^{\circ}$ año reconoce la participación de ambos, pero enfatiza que los que promovían por general este diálogo eran los españoles, como se puede ver en el párrafo siguiente:

En la gran mayoría de los casos, los parlamentos fueron iniciativas generadas y financiadas por los españoles. En ellos, cada parte expresaba su postura a través de largos discursos. Los oradores indígenas aprovechaban la oportunidad para quejarse de los abusos españoles, al mismo tiempo que realizaban propuestas para mejorar las relaciones. Por su parte, los oradores españoles se comprometían a respetar los acuerdos de paz (Zig-Zag, 2012a:58).

La imagen de los indígenas como desobedientes al orden y resistentes se traslada a los relatos sobre la historia del siglo XIX, esto pese a lo escaso de temáticas que incorporen a estos grupos en el currículum del curso. El texto de $6^{\circ}$ año nombra o desarrolla temáticas asociadas a los indígenas casi de manera excepcional, sin embargo las incorporaciones que se hacen en el relato relaciona a los indígenas a actividades violentas como muestra el siguiente párrafo "Las montoneras realistas tuvieron el apoyo de muchos pequeños campesinos de la región, especialmente de las comunidades indígenas pehuenches que habitaban la zona" (Zig-Zag, 2012b: 56).

Es interesante destacar que el relato que se construye sobre el carácter guerrero y rebelde del indígena no aparezca reafirmado en uno de los contenidos más contingentes como lo es la ocupación de La Araucanía. En el abordaje de este contenido el texto opta por una especie de discreción que parece busca evitar el carácter conflictivo del contenido a abordar. El texto sobre el tema de la ocupación de La Araucanía indica que:

\section{El Estado tomó la decisión de integrar a su dominio ese importante territorio. Para llevarlo a cabo se preparó un plan militar, ideado por Cornelio Saavedra, quien avanzó militarmente por la zona y posteriormente, fundó ciudades. Así se originaron Lebu, Mulchén y Temuco. \\ En 1881, durante la Guerra del Pacífico, Gregorio Urrutia reinició con fuerza el proceso que terminaría con la derrota de los mapuches en 1883 (Zig-Zag, 2012b:110).}

Finalmente el texto de $6^{\circ}$ año básico para evitar cualquier conflicto indica que la ocupación de los territorios mapuches traerá consecuencias que se extienden hasta la actualidad para este grupo.

Como hemos visto los textos de historia de Chile para la educación básica transmiten en sus contenidos una representación del indígena asociada a un carácter guerrero y rebelde, resaltando que este comportamiento no responde a una respuesta ante la conquista de los españoles, sino que se insiste en que responde al desarrollo cultural del indígena. Lo anterior de acuerdo con los textos trajo consecuencias en cuanto significó un obstáculo para la pervivencia de los españoles en el territorio y convirtió a Chile en una zona de conflicto permanente por lo menos en lo que concierne al período de conquista y colonial. De esta manera los textos de estudio de historia de Chile consolidan prejuicios y estereotipos relacionados con la representación del indígena como un ser bárbaro y violento.

\section{Conclusiones}

Nuestro objetivo al revisar los textos de estudio de historia de Chile utilizados en la educación 
básica fue conocer ¿cuál es la presencia que tienen los indígenas en los textos de estudio de historia de Chile para la educación primaria? Y ¿cómo son las narraciones sobre los indígenas en los textos de estudio de historia de Chile para la educación primaria? Respecto de la primera interrogante hemos podido establecer que los contenidos referidos a los indígenas se encuentran presentes para los tres cursos en los que se tratan las temáticas de historia de Chile en la educación básica, esto es una confirmación de lo que ya habíamos observado en una investigación anterior referida a la presencia de los otros y las otras (Villalón y Pagès, 2013). La revisión también nos muestra que de manera similar a lo ocurrido en las revisiones realizadas para el caso de la enseñanza de los indígenas en Estados Unidos (Shear, et al., 2015), los textos en Chile instalan los contenidos referidos a los indígenas en el pasado lejano anterior al 1900, para luego hacer invisible la presencia de los indígenas en la historia reciente. Los textos de estudio en Chile también asocian la inclusión de los indígenas con conceptos como pueblos originaros, aspectos folklóricos o la violencia y comportamiento incivilizados.

La confirmación de la presencia de los indígenas en los tres textos de estudio revisado nos permite plantearnos que el debate sobre la presencia de los otros y las otras (como mujeres, niños, trabajadores, homosexuales, entre otros) en la enseñanza de la historia debe considerar avanzar hacia el estudio de cuáles son los discursos que se construyen sobre estos actores, ya que su presencia entre los contenidos de los currículos de historia es una realidad.

Con relación a la segunda pregunta que nos planteamos podemos establecer que las representaciones que se hacen sobre los indígenas se caracterizan por un relato que los asocia al proceso de mestizaje y el legado cultural representado en la tradición o folklore nacional. Un segundo relato es el que representa al indígena con un carácter guerrero y rebelde, resaltando que este comportamiento no responde a una respuesta ante la conquista de los españoles, sino que es resultado del desarrollo cultural del indígena. Las representaciones sobre los indígenas que construyen los textos de estudio no se alejan de los discursos que se han identificado en otras investigaciones en Estados Unidos y México en las que las narraciones profundizan estereotipos sobre los indígenas y consolidan discursos asociados al carácter salvaje o folklórico.

\section{Agradecimientos}

Convenio de Desempeño UTA 1309 "Educación de calidad para tod@s: un compromiso con la formación inicial de profesores de la Universidad de Tarapacá".

\section{Referencias Citadas}

Arcos Vázquez, C.

2010 Bajche' mi ik'ejlel wokolol tyi wajali tyi laklumal. Normas, conductas y orden en los pueblos originarios [Una perspectiva histórica]. Programa de Fomento al Desarrollo de las Ciencias y Artes Mayas y Zoques (FOCAMAZ). Sna' Jtz'ibajpm, Cultura de los Indios Mayas, AC. Universidad Intercultural de Chiapas UNICH. San Cristobal de las Casas, Chiapas.

Benejam, P. y Pagés, J.

1997. Enseñar y aprender Ciencias Sociales. Geografía e Historia en la Educación Secundaria. Barcelona: ICE de la Universidad Barcelona/Horsori.

Carretero, $\mathrm{M}$.

2007 Documentos de identidad: la construcción de la memoria histórica en un mundo global. Buenos Aires: Paidós.

Dosil Mansilla, J.

2010 La construcción de las identidades en México, ¿y los indígenas? En Pagès, J. y González, N.(Coords). 2010. La construcció de les identitats i l'ensenyament de les Ciències Socials, de la Geografia i de la Història . Univ. Autònoma de Barcelona.
Fixico, D. L.

1998 Ethics and responsibilities in writing American Indian history. En D. A. Mihesuah (Ed.), Natives and academics: Researching and writing about American Indians pp. 84-99. Lincoln: University of Nebraska Press.

Lévesque, $\mathrm{S}$.

2008 Thinking historically: Educating students for the twenty-first century. Toronto: University of Toronto Press. Mesbo, Jo.

2015 El Ratpenat. Barcelona. Editorial Proa.

Pagès, J.

1994 La didáctica de las ciencias sociales, el currículum y la formación del profesorado. En Signos. Teoría y práctica de la educación, (13), pp. 38-51.

Santillana Editorial

2012 Texto del Estudiante. Historia, Geografía y Ciencias Sociales, Segundo año Básico. Santiago, Editorial Santillana Santisteban, A. y Pagès, J.

2011 Didáctica del conocimiento del medio social y cultural en la educación primaria: ciencias sociales para comprender, pensar y actuar. Editorial Síntesis. 
Shear, S. B.; Knowles, R. T.; Soden, G. J. y Castro, A. J.

2015 Manifesting Destiny: Re/presentations of Indigenous Peoples in K-12 US History Standards. Theory \& Research in Social Education, 43(1), 68-101.

\section{Urrieta, L.}

2004 Dis-connections in "American" Citizenship and the Post/ neo-colonial: People of Mexican Descent and Whitestream Pedagogy and Curriculum. Theory \& Research in Social Education, 32(4), 433-458.
Villalón, G., y Pagès, J.

2013 ¿Quién protagoniza y cómo la historia escolar?: La enseñanza de la historia de los otros y las otras en los textos de estudio de Historia de Chile de educación primaria. Clio \& asociados: La historia enseñada, (17), 119-136.

\section{Zig-Zag Editora}

2012a Historia, Geografía y Ciencias Sociales. Texto del Estudiante, Quinto año Básico. Santiago, Editorial Zig-Zag. 2012b Historia, Geografía y Ciencias Sociales. Texto del Estudiante, Sexto año Básico. Santiago, Editorial Zig-Zag. 